\title{
A Survey and Analysis on the Current Status of the Mobile Applications for Garden Design
}

\author{
Hyun-Ji Kim, Kyoung-Youn Lee, Yu-Jin Song, Yi-Seul Joo, and Kyung-Mee Lee* \\ Gardens and Education Division, Korea National Arboretum, Pocheonsi 11186, Korea
}

\begin{abstract}
This study aimed to examine the current status of mobile applications for garden design and extract valuable contents for the development of garden design programs for future reference. Mobile applications released in Korean or English on Google Play App Market as of July, 2018 were analyzed in this study (1 application in Korea and 14 applications overseas). The purposes of this study are to analyze the components of the programs for those who actually intend to create a garden and to use it as a resource for developing mobile applications for garden design. Thus, program components and contents were analyzed for garden design applications based on real space ( 1 application in Korea and 3 applications overseas) that could actually help users. The analysis of mobile applications for garden design shows that while overseas garden programs are rapidly developing in various fields, the number of garden design mobile applications developed in Korea and the amount of information platforms are significantly insufficient. This study suggested flowchart for garden design mobile applications based on the analysis results of existing garden design application. This flowchart includes a series of processes from planning/designing gardens to purchasing plants and facilities to constructing/maintaining gardens for users who intend to design and create a real garden. Furthermore, this study proposed a freemium business model based on 4R(Reflex, Reality, Real place, Real communication) marketing strategies for mobile applications. Realistic experiences can be increased through graphics and information about gardens and plants provided in this study, and location-based information services as well as the creation of systems connected with vendors and suppliers of products related to gardens can induce consumers' purchasing behaviors. Additionally PR activities through various garden-related cultural events are expected to attract more users.
\end{abstract}

Keywords: garden application, garden design mobile application, garden design program

\section{Introduction}

Since the Act on the Creation and Furtherance of Arboretums and Gardens (hereinafter, the Garden Act) was enacted in July, 2015, people's demand for and interest in the promotion of garden culture have increased (Lee et al., 2017), and various garden expos and shows such as the International Garden Exposition Suncheon Bay Korea and the Korea Garden Show have been held annually as part of the government's policy to promote garden culture. The recent spread of garden culture and increase in leisure time have also raised people's interest in and preference to nature and plants (Song and Kim, 2012), and various types of gardens have been created and operated by individuals or organizations. 
Interest in gardens in society has increased and garden culture has been spread, which is expected to lead to the development and consumption of garden designs (Yum and Joo, 2017). While citizens' interest in gardens has increased, most of the educational programs on gardens currently available for them focus only on the maintenance and management of plants such as plants themselves and indoor pot plants. It is indeed difficult for those who intend to design and create their own garden to obtain information on the creation of gardens from these limited educational programs. Of course, education on garden design is provided as a special programs, but as schedules, locations and the number of allowed participants are limited, it is slightly difficult to provide comprehensive services for citizens. In order to create and spread garden culture in the long term, garden culture services need to be expanded to ensure people conveniently access information they need based on their interest.

To ensure citizens design and obtain information on gardens in an easier and more convenient way, it is necessary to provide garden service programs that are accessible by anybody at anytime and anywhere. As iPhones started to be imported into Korea in late November, 2009, the domestic smartphone market has been expanded and the demand for applications has also rapidly increased (Kwak and An, 2013). Recently, a variety of applications that provide practical convenience plays a role as a personal agent for consumers (Lee, 2013). Mobile applications ${ }^{1}$ ) affect the lifestyle of people in modern society in various areas, including not only games but also SNS, education, healthcare, music and art. In areas associated with gardens, mobile applications have recently been developed and operated based on smart systems that integrate ICT (Information and Communication Technology) and IoT (Internet of Things). Applications provide a wide range of data and actual learning environments through asynchronous interactions and simulations that transcend space, and social communication structures through SNS, and thus allow learners to enjoy various learning opportunities regardless of geographical and temporal constraints (Lee, 2011). Practical effects among the public can be obtained by providing garden services through applications based the educational usefulness of applications in this age of smartphones. These applications are expected to provide more practical help for those who are interested in gardens, in particular, those who plan to create gardens to design their own gardens and purchase necessary plants and materials.

Against this backdrop, this study aimed to examine the current status of mobile applications for garden designs that have been developed in Korea and abroad, to extract contents essential for garden design programs, and thus to provide basic data that can be utilized in developing mobile applications for garden design going forward.

\section{Subjects and Methods}

The purposes of this study were to understand the characteristics of mobile applications for garden design that have been developed in Korea and abroad, to analyze their types and compositions, and thus to identify components required for mobile applications and additional contents. In line with that, among mobile applications that were registered on Google Play APP Market in Korea and abroad as of July, 2018 (Google Play Store, n.d.), free programs released either in Korean or English were surveyed and analyzed. A complete enumeration survey was performed on applications related to gardens, and they were divided into 5 types (Figure 1).

The first type included 'programs for garden design' in which users can plan and design gardens, and draw a ground plan or a 3D design, and the second type included 'programs for garden and plant management' that provide information

1) An application, as an application software program, means broadly all software programs run on an operating system (OS), and narrowly a software program that a user directly uses in an OS (Yang and Lee, 2011). A mobile application means a content software program run on a mobile device such as smartphones, and users consume mobile applications after downloading them (Song, 2012). 
on the growth and characteristics of plants and the cycle of maintenance or suggest suitable plants for different seasons, climatic conditions and regions. The third type included 'programs for garden education' provided for preschoolers and children, and the fourth type included 'programs for garden image' that provide ideas for and images of gardens with various themes. The fifth type included 'programs for garden games' that have themes such as tending gardens and cultivating plants. Apart from these five types, different types of applications related to gardens have been developed and used such programs to give information on individual gardens, and those to identify plants.

One of the purposes of this study was to extract essential contents for garden design programs, and thus, this study focused on 'programs for garden design' out of the five types of programs. A complete enumeration survey was conducted on mobile applications about garden design that were released in Korea and abroad, and it was found that there were one mobile application in Korea and 14 mobile applications abroad as of July, 2018, and they were divided into three types as follows: design programs based on real space, design programs based on virtual space, and garden design and plan text books (Figure 2).

'Mobile applications for garden design based on real space' allow users to directly designate the area and size of gardens and to divide the area, or to design gardens using satellite pictures designated by users or those taken by users as a background. 'Mobile applications for garden design based on virtual space' allow users to arrange plants and garden facilities using spaces or grids arbitrarily given by the programs or gardens designated by the programs as a background. Unlike those based on real space using real-world scale, most programs based on virtual space are composed of simple images,

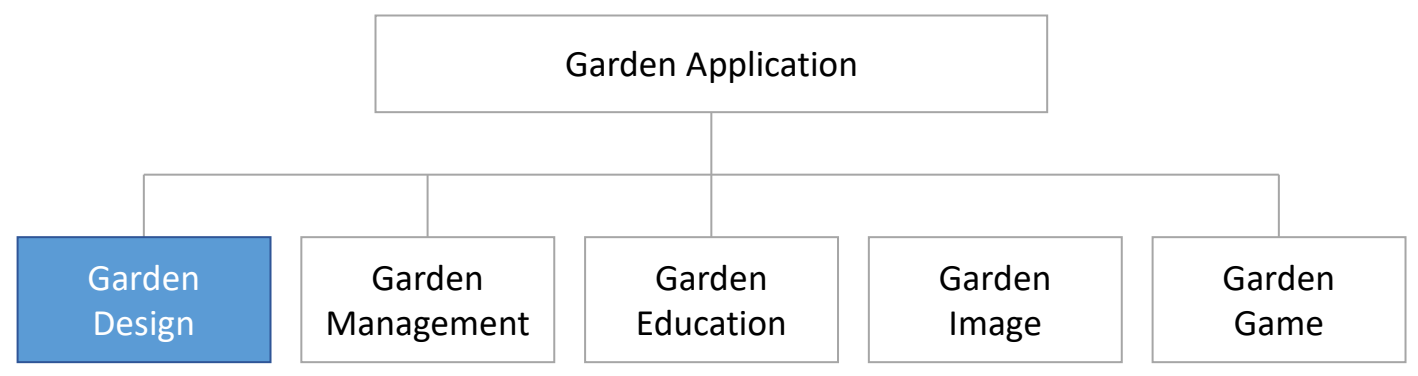

Figure 1. Garden application types.

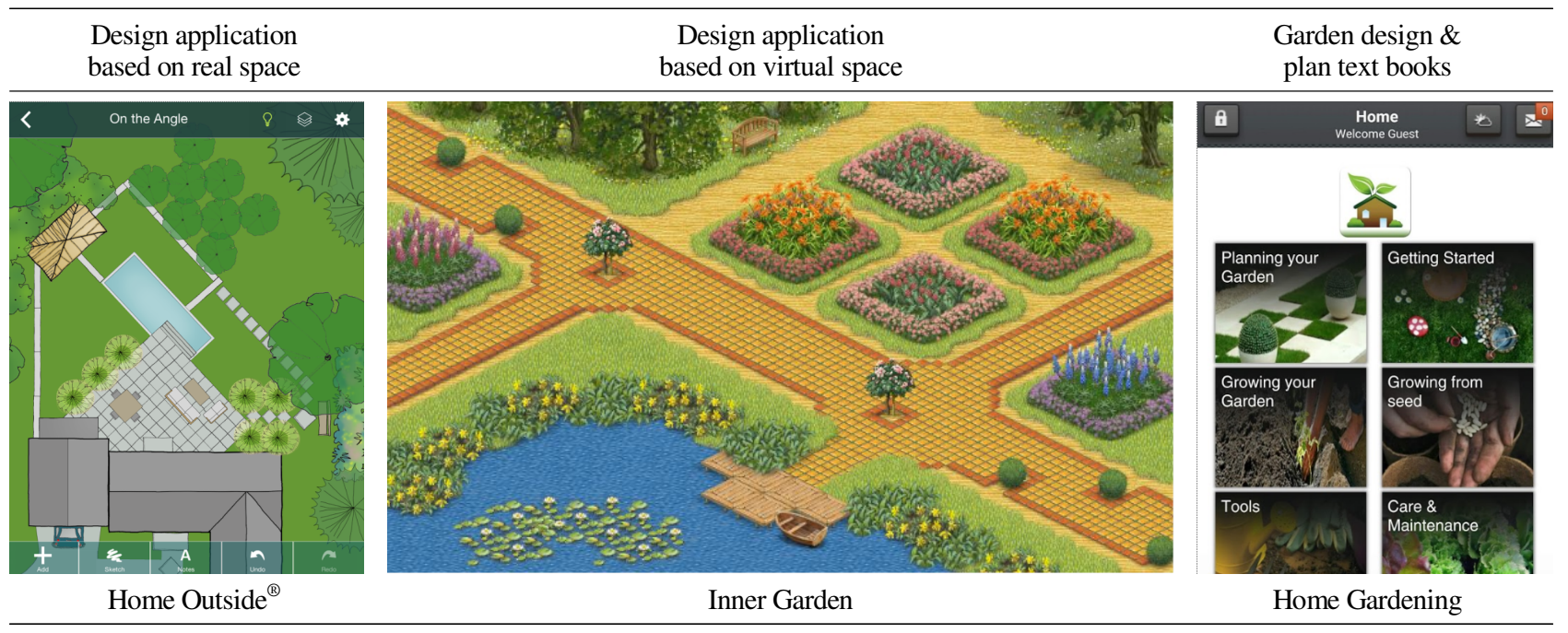

Figure 2. Three types of garden design mobile application. Images were reprinted from Google Play (retrieved from https://play.google.com/store/apps). 
and thus it is difficult for users to plan and design gardens as they wanted. 'Garden design and plan text books' are to provide information required not for designing gardens, but for creating and managing gardens such as garden plans and designs, information on plants and tips for gardening (Table 1).

As this study aimed to analyze components of programs for those who actually intend to create gardens and to provide information that can be utilized in developing mobile applications for garden design going forward, this study focused on 'mobile applications for garden design based on real space' that can provide practical help for users, and their components and contents were analyzed. In addition, designing gardens is a process of identifying the characteristics of individual plants, combining them to be harmonized and finding the optimal spots for individual plants (Park, 2010). For this reason, the selection of plants and planting designs are an essential part of gardens, and people's interest in and the importance of planting flowering plants have recently increased (Song and Kim, 2012). In this regard, contents about planting that were included in programs for garden design were also analyzed to identify components required to develop mobile applications for garden design going forward.

\section{Results and Discussion}

\section{Analysis of mobile applications for garden design based on actual space}

\section{Domestic mobile application for garden design — Indoor Garden Design}

Indoor Garden Design (Figure 3), the only mobile application for garden design released in Korea, was developed by the Rural Development Administration, and users can mix pots, plants and materials as they want and arrange them in an indoor space to create an indoor garden. The detailed components of the application are as shown in Figure 4. Users can use images of indoor spaces provided by the program, or insert those taken by themselves as a background. The program provides information on 70 plants that can be utilized in designing gardens, and information on the use of a total of 314 plants (basic information on indoor plants and information on management) is also available.

As the application was developed by the Rural Development Administration based on research data, technical information on plants (growth characteristics and maintenance information) is provided. Plants can be classified based on their description, and images of real plants are utilized as a source of the application. In addition, 8 types of videos on how to create indoor gardens are provided for users to more easily create indoor gardens, and the homepage of the Rural Development Administration is linked with the application, offering latest news to provide more practical information for those who plan to create an indoor garden and grow indoor plants.

Table 1. Garden design application list

\begin{tabular}{|c|c|c|c|}
\hline Category & & Application & Total \\
\hline Domestic & Based on real space & Indoor Garden Design & 1 \\
\hline \multirow[b]{3}{*}{ International } & Based on real space & Home Outside ${ }^{\circledR}$, VR Gardens, PRO Landscape Home & 3 \\
\hline & Based on virtual space & Garden Squared, Planter-Garden Planner, Inner Garden, Yates My Garden & 4 \\
\hline & $\begin{array}{l}\text { Garden design \& } \\
\text { plan text book }\end{array}$ & $\begin{array}{l}\text { Garden Landscape Design, Free Gardening Tips, } \\
\text { Garden Square Vegetables Guide, Home Gardening, } \\
\text { Landscape \& Garden Calculators, Organic Gardening(Applause Apps), } \\
\text { Organic Gardening(Sun Media Soft) }\end{array}$ & 7 \\
\hline
\end{tabular}

Note. One domestic application and 14 international applications were analyzed. 


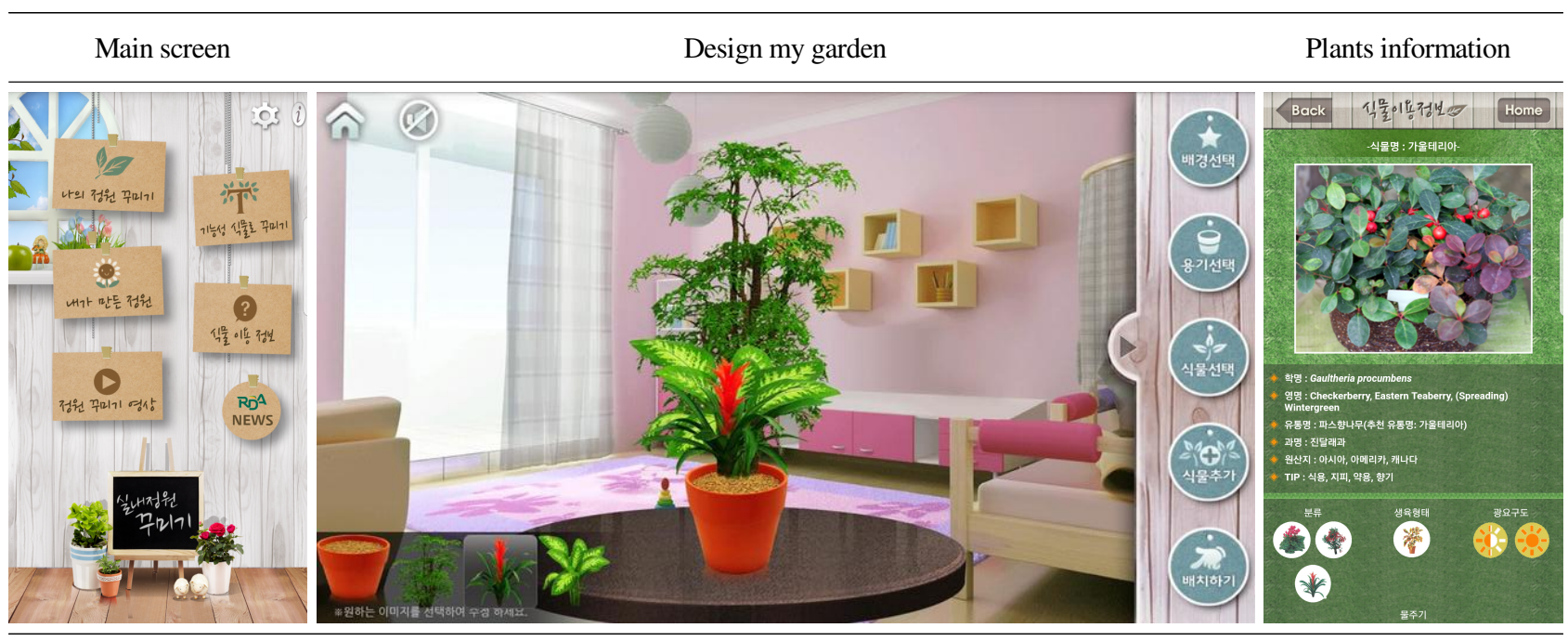

Figure 3. Indoor Garden Design application design. Images were reprinted from Google Play (retrieved from https://play.google.com/store/apps).

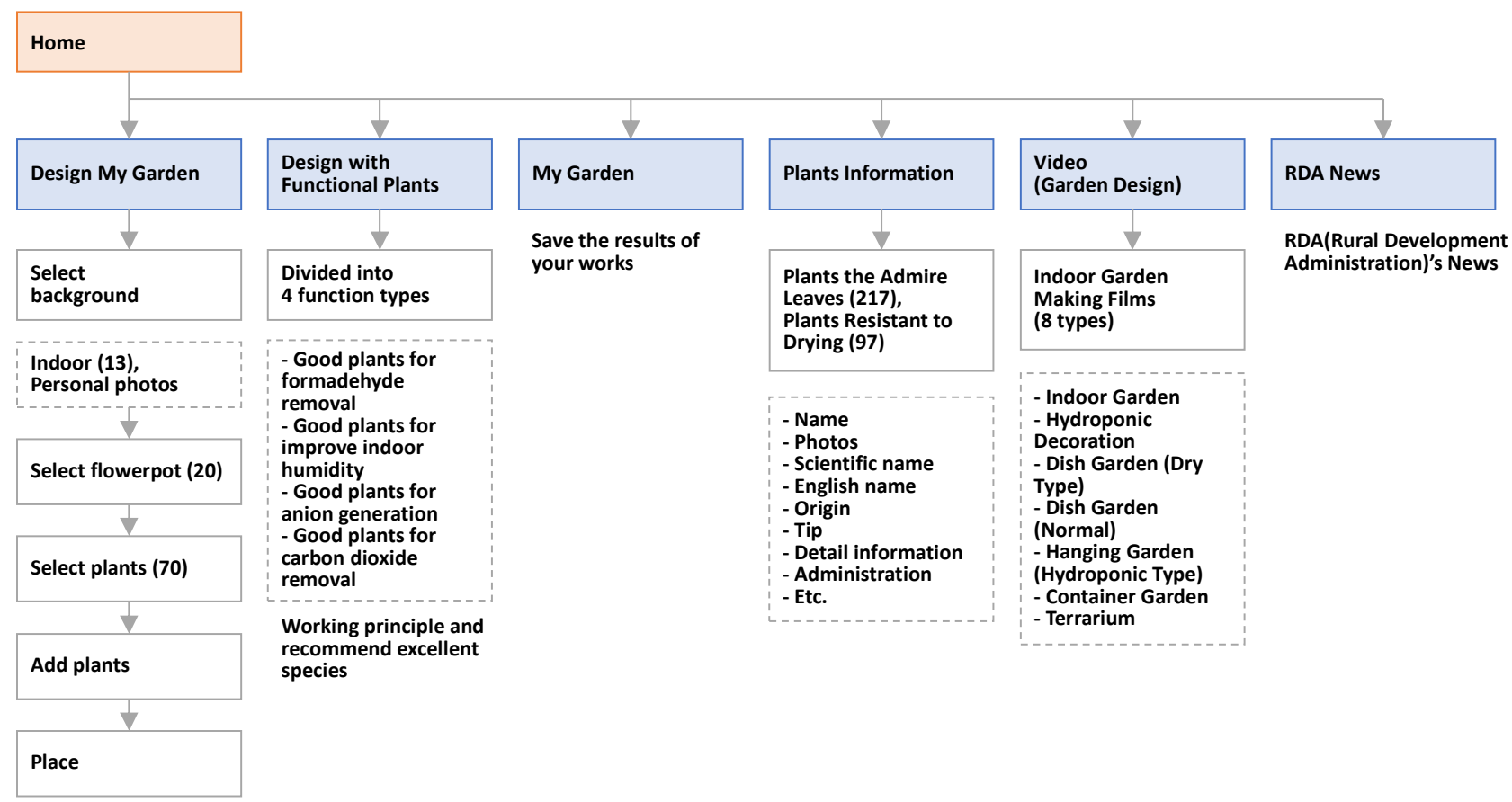

Figure 4. Flowchart of Indoor Garden Design application.

\section{Overseas mobile application for garden design - Home Outside ${ }^{\circledR}$}

Home Outside ${ }^{\circledR}$ (Figure 5) is a mobile application released overseas. Users can insert floor plans they drew themselves or satellite images, and select a certain area. Based on the selected area, they can design floor plans for gardens. As various floor plan samples are provided, those who are not familiar with designing ground plans can easily understand how to design gardens and use the application. Plants are divided into trees, shrubs, flowering plants and grass, and drawing images regardless of species of trees are provided. As the mobile application is designed for outdoor gardens, it also provides other contents such as structures (buildings, stairs, parking areas, pergolas, fences), artificial elements (patios, sidewalks, walls, stones), furniture for gardens and other facilities. The detailed components are as shown in Figure 6. 


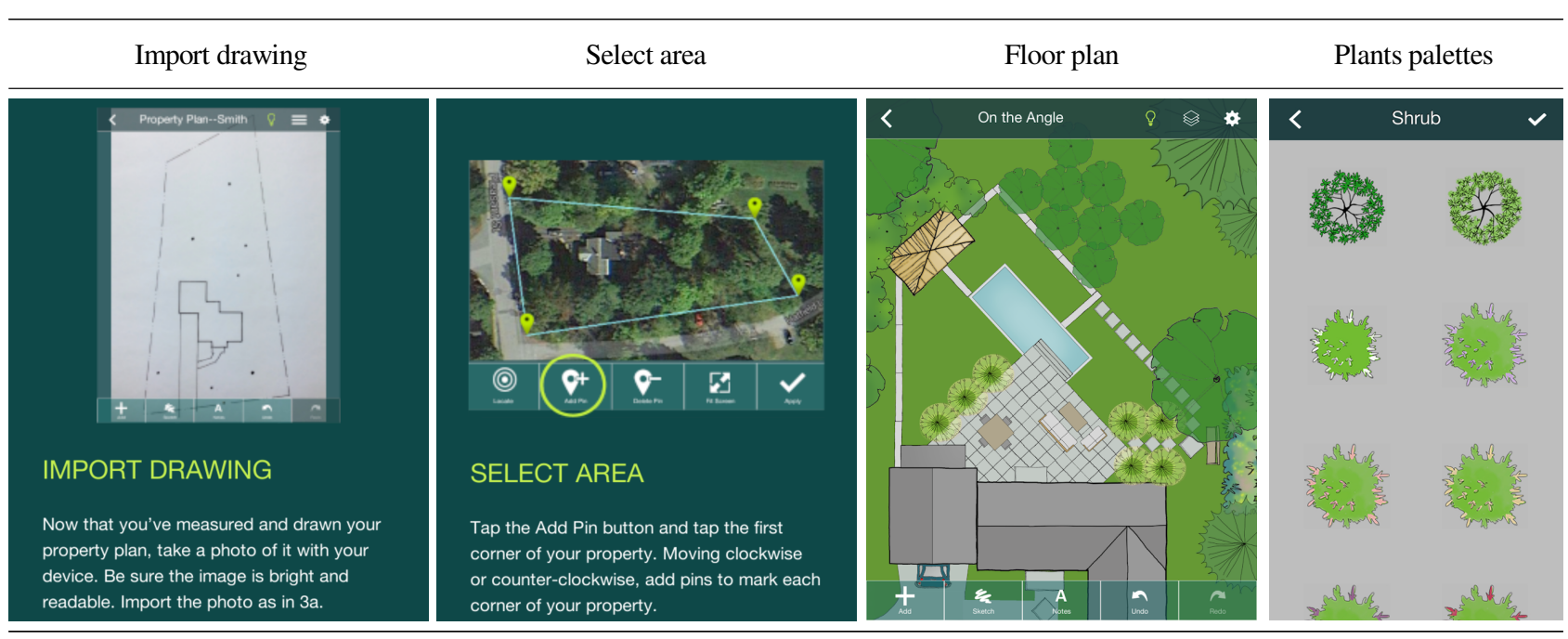

Figure 5. Home Outside ${ }^{\circledR}$ application design. Images were reprinted from Google Play, (retrieved from https://play.google.com/store/apps).

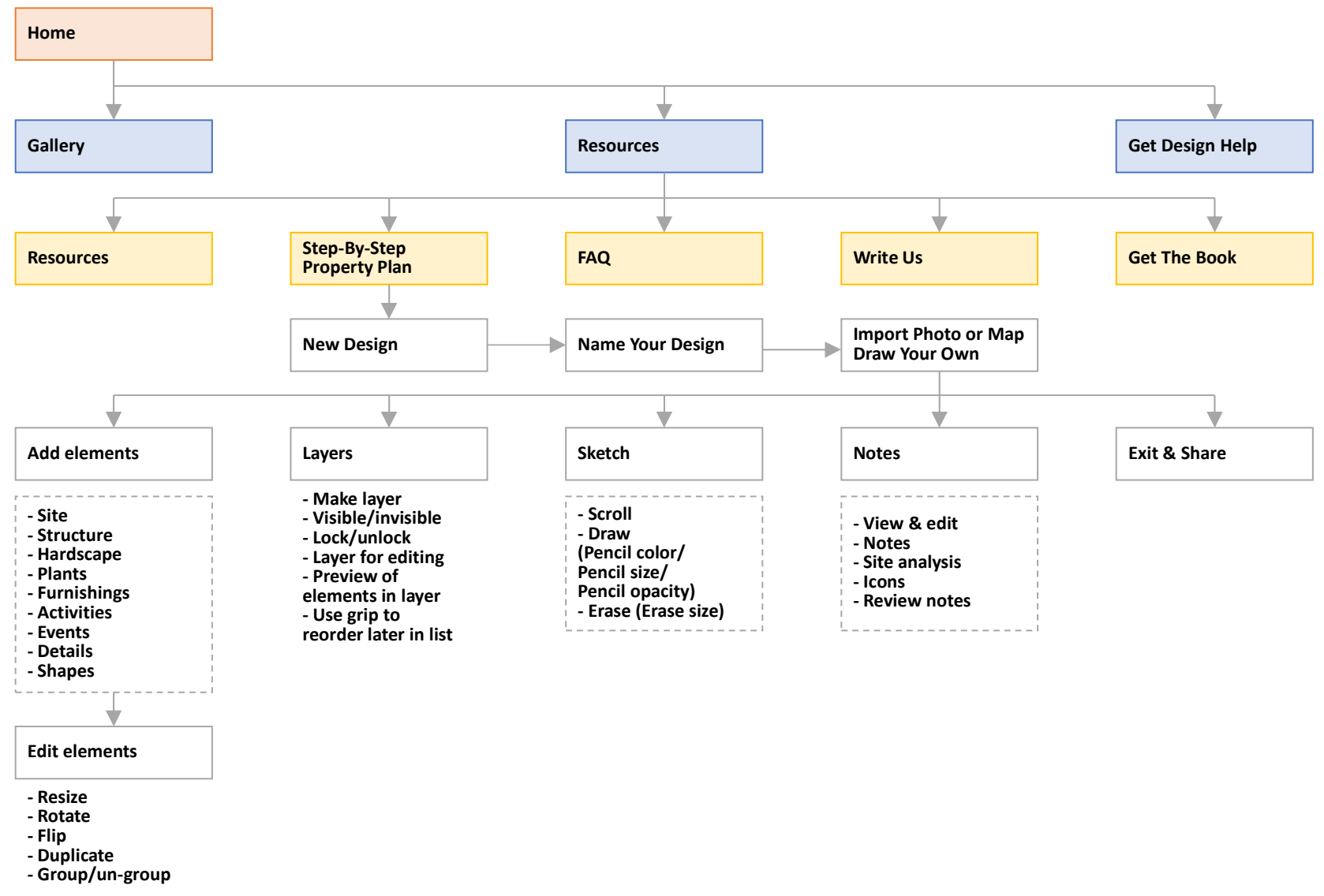

Figure 6. Flowchart of Home Outside ${ }^{\circledR}$ application.

Home Outside ${ }^{\circledR}$ applies the function of Photoshop to ensure each item can be arranged as a layer, and items can be grouped together, which can be conveniently used when drawing floor plans. As the function of memos is also included, users can take notes on any information on each item, which allows them to write the name of plants on plant icons of which species are not separately identified, or to write the planned layouts or characteristics of arranged facilities or pavements. 


\section{Overseas mobile application for garden design - VR Gardens}

VR Gardens (Figure 7) is a mobile application that utilizes VR technology in designing gardens. The process of designing gardens as follows: setting the location of a garden - dividing into grids - marking the points of the compass - setting entrance - setting garden type - designing plants and facilities. Using the application, the planned garden can be viewed from any angle of 360 degrees, not only as a floor plan, but also as 3D view, Eye view and VR view, allowing users to more easily imagine the images of their planned gardens and design their space. The details are as follows (Figure 8).

Users can enter the address of the planned garden and select Google satellite images as a base, or set the size of their planned garden and the points of the compass to create a boundary and to use it as a base without designating a certain location. Gardens are divided based on their purpose into Classic, Courtyard, Easy care, Kids \& Family, Kitchen and Modern types, and each type provides 5 basic frames, and users can also build their own (Build your own) frame.2) Graphic image

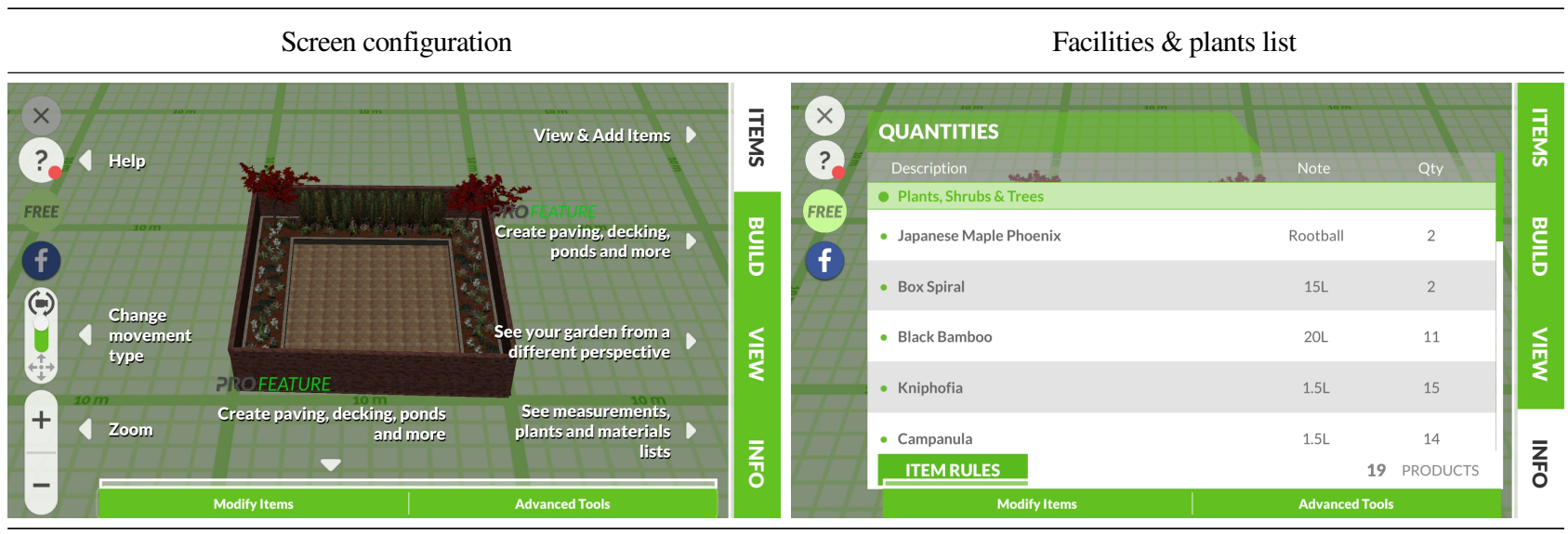

Figure 7. VR Gardens application design. Images were reprinted from VR Gardens application.

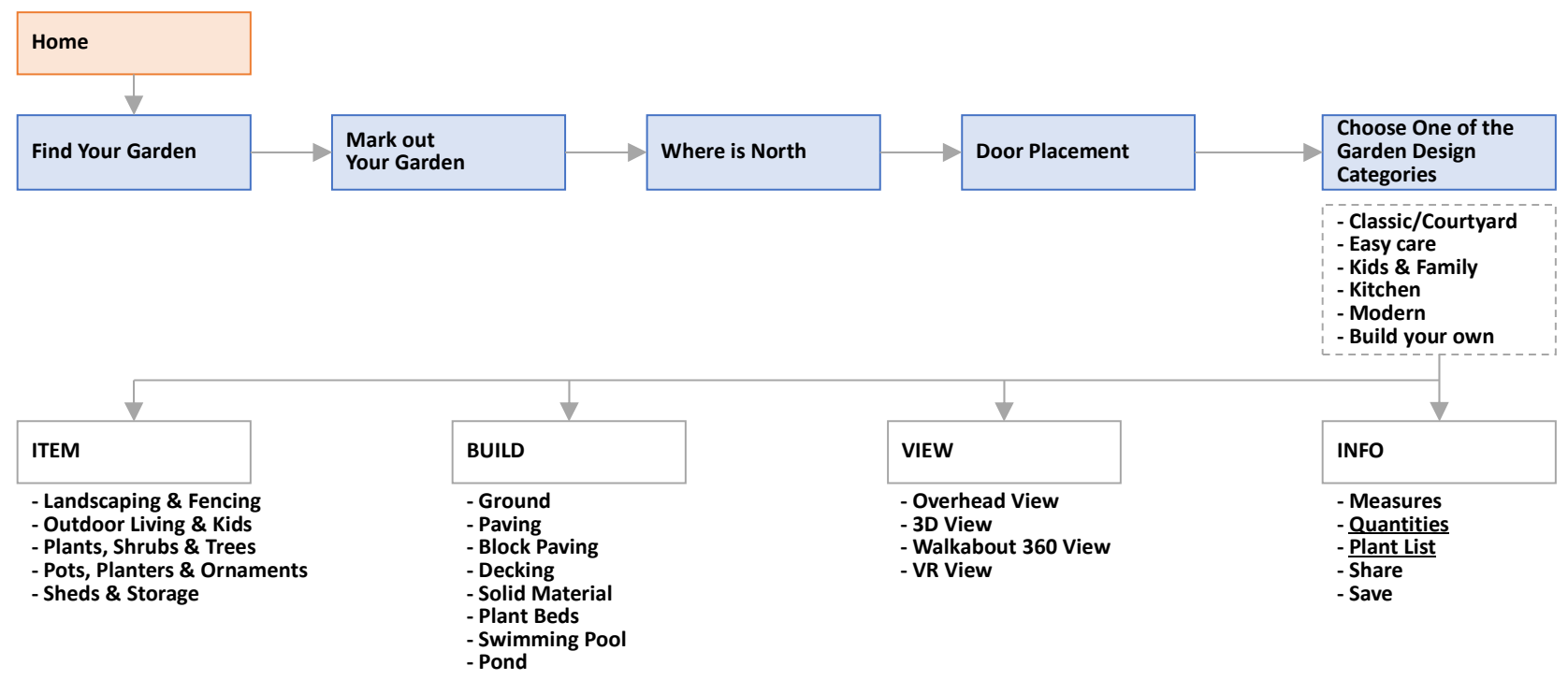

Figure 8. Flowchart of VR Gardens application.

2) VR Gardens is available as the free version, plus version and pro version. The free version provides only two types of gardens, one basic frame for each type and 5 gardening items (facilities and plants). Users can buy either the plus version or the pro version depending on their needs to design their own gardens based on various concepts and items. 
sources for different species of plants are provided, and other garden facilities such as boundaries and fences, outdoor living, planter and sculpture, bicycle racks and warehouses are provided as a content.

After designing a garden, the total list of plants and facilities added to the planned garden is provided for users to view them at one view, and different images of the planned garden depending on weather conditions and time are also available. Using this function, users can determine the species and location of plants considering the sunny and shady spots. They can also imagine the real and detailed views of their planned garden based on various views and changing views depending on weather conditions and time.

\section{Overseas mobile application for garden design — Pro Landscape Home}

Pro Landscape Home (Figure 9) is an application in which users can arrange plants and facilities using photos that they took themselves as a background, and allows those who are difficult to understand floor plans to insert images of real objects onto photos they choose and thus to more easily design gardens unlike other floor plan-based programs that require the understanding of space. This program provides several categories of contents including trees, shrubs, flowering plants, ground cover plants, pavements, others and lighting, and a list of items required to created the planned garden (including plants and facilities) after completing designing. The details of the application are as follows (Figure 10).

Contents about plants use the name and images of real plants as a source, and when users select the climatic zone where they live, the list of suitable plants for the climatic zone is displayed. Pro Landscape Contractor developed by the same company is for landscaping experts who can adjust the price, size and quality of items required to create their designed garden, and issue a quotation based on the data. Using this program, contractors can discuss designs and quotations during meetings with clients in a short period of time, which can be practically used.

\section{Analysis of the components of mobile applications for garden design and contents on plants}

\section{Analysis of the components of mobile applications for garden design}

Based on the analysis results of 4 types of mobile applications above, 2 components that were commonly observed were obtained. The first component for designing gardens was 'base.' Bases were provided in the forms of satellite map images of the spaces that users designated or floor plans (users can draw outlines based on their target sites) or photos of target

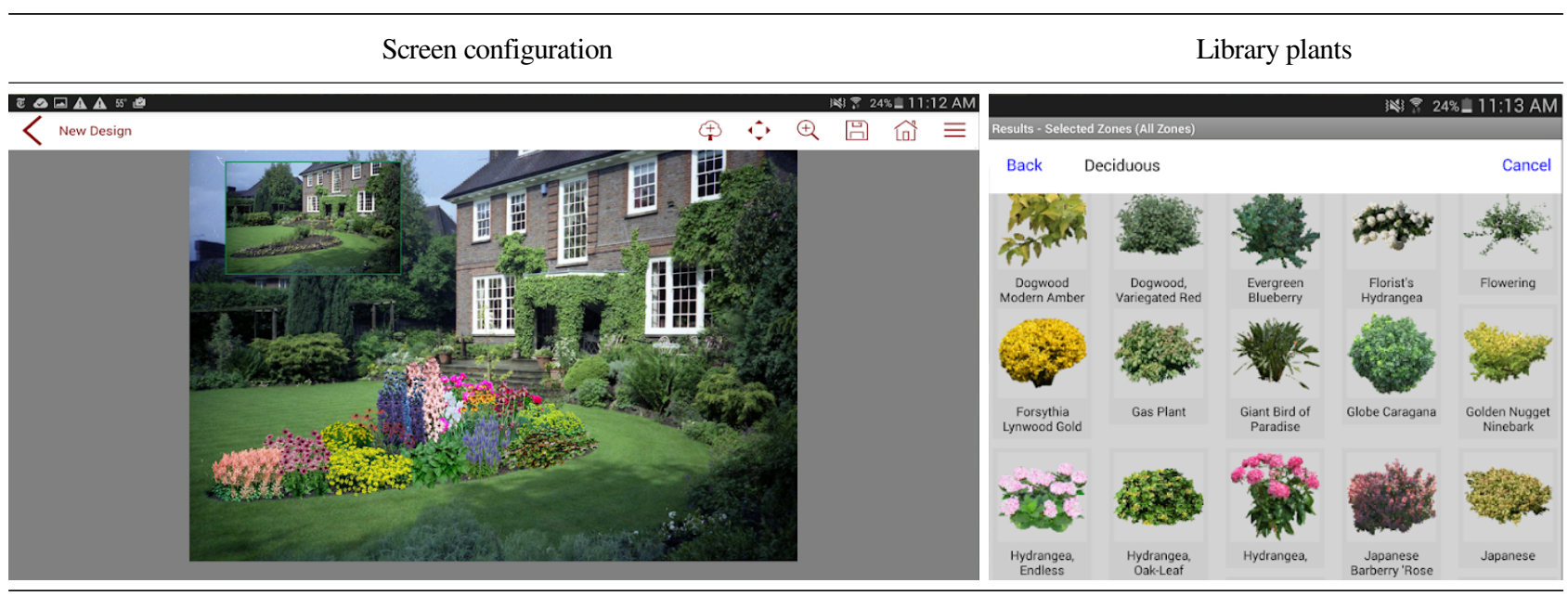

Figure 9. Pro Landscape Home application design. Images were reprinted from Google Play (retrieved from https://play.google.com/store/apps). 


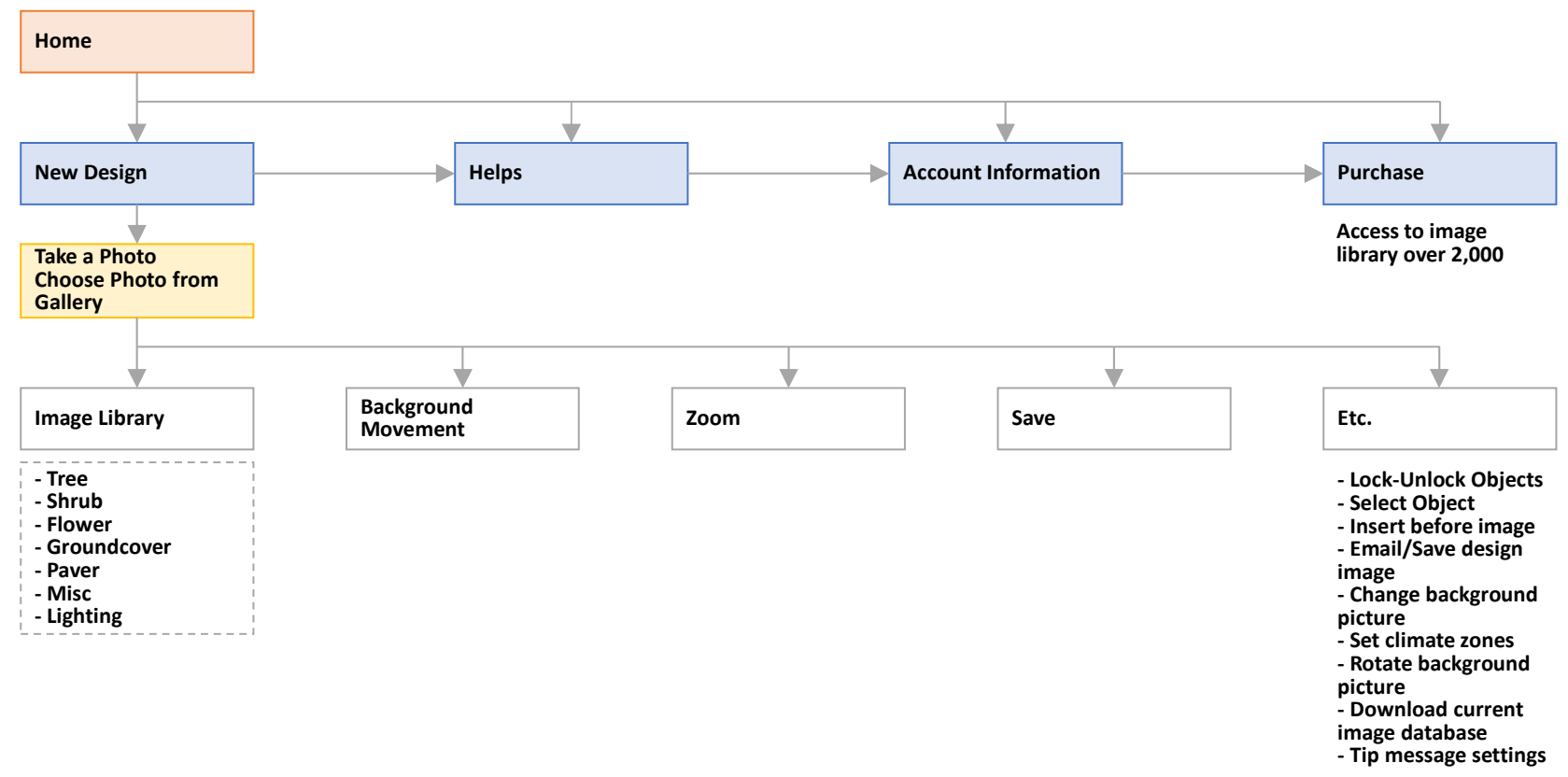

Figure 10. Flowchart of Pro Landscape Home application.

sites they took. The second component was 'elements for garden design' including plants, structures, facilities, sculptures, pavements and lighting, and they were provided in the forms of floor plans, elevation designs or 3D pictures or photos. Based on the 'bases' they want to design, users can arrange 'elements for garden design' in the desired size, number and form on any place.

Apart from the two commonly observed elements, the components of each application were analyzed to identify additional elements required for mobile applications for garden design such as Indoor Garden Design's information on plants (growth characteristics and maintenance information) and videos on th creation of gardens, Home Outside ${ }^{\circledR}$,s samples of garden floor plans and memo function, VR Garden's variety of view images depending on weather conditions and time and list of construction items, and PRO Landscape Home's system to select species of plants suitable for the climatic zone where users live, list of construction items, and estimated quotations.

The currently available mobile applications for garden design arrange design elements on bases, and thus are suitable to write basic plans for garden designs. However, they provide not enough practical information (suitable species of plants, plants and facilities required to create the designed gardens, budget, suppliers, maintenance methods, advices from experts) for those who actually plan to create gardens. In order to develop practical mobile applications for garden design for those who actually plan to create gardens based on the components of the currently available applications, it will be necessary to consider a series of processes from 'preparation' to 'planning and designing gardens' to 'creating gardens' to 'maintaining gardens' all together.

In the first stage of preparation, overall knowledge and information on the creation of gardens are obtained including garden images of different garden types, guidelines for design methods and considerations, and basic information on plants and materials for gardens. They can be provided in forms of sample images and textbooks by mobile applications. In the second stage of planning and designing gardens, garden design elements (plants, facilities, sculptures, lighting, irrigation systems, etc.) are drawn or arranged on bases as the existing applications showed. In addition, it will be possible to provide more practical help for those who plan gardens by suggesting suitable species of plants for the climatic conditions 
of the target areas of gardens, and by sharing design plans with other users to establish networks and information exchange systems between users. In the stage of creating gardens, it will be possible to provide a list of plants and facilities required to create the gardens designed by users, to write the estimated quotations for creating the gardens, and to establish systems linked with purchasing sites or suppliers of plants and related facilities, although it is difficult to directly access to the information due to the characteristics of applications. In the fourth stage of maintaining gardens, tips for maintaining planted plants (watering, soil, harmful insects, cultivation and harvest time, etc.) and counselling with experts can be provided for users to find answers to questions they might have while maintaining real gardens.

\section{Analysis of contents about plants in mobile applications for garden design}

Contents about plants in mobile applications for garden design as a design element were analyzed ( 8 applications ${ }^{3)}$ ), and it was found that they were largely divided into two categories (design interface, plant information). Information provided by plant design interfaces can be in turn divided into methods of expression (design, species of trees) and classification (description, shape, growth characteristics, others). Information on plants included the characteristics of plants, cultivation and harvest time, and maintenance methods (Table 2).

The domestically-developed program was designed to create indoor gardens, and thus its interface was composed of the elevation images of a total of 70 species, and provided information on the characteristics of plants and methods for maintaining plants. Those developed overseas provided various plant designs, while only 3 out of a total of 7 programs establi-

Table 2. Contents of plants in garden design applications

\begin{tabular}{|c|c|c|c|c|c|}
\hline \multicolumn{4}{|l|}{ Category } & \multirow{2}{*}{$\begin{array}{c}\text { Domestic } \\
-\end{array}$} & \multirow{2}{*}{$\frac{\text { International }}{3}$} \\
\hline \multirow{10}{*}{$\begin{array}{l}\text { Plants } \\
\text { design } \\
\text { interface }\end{array}$} & \multirow{6}{*}{$\begin{array}{l}\text { Method of } \\
\text { expression }\end{array}$} & \multirow{3}{*}{ Design } & Flat surface & & \\
\hline & & & Aspect & 1 & 3 \\
\hline & & & $3 \mathrm{D}$ & - & 1 \\
\hline & & \multirow{3}{*}{ Species of trees } & Divide & 1 & 3 \\
\hline & & & Not divide & - & 2 \\
\hline & & & Mix & - & 2 \\
\hline & \multirow{4}{*}{\multicolumn{2}{|c|}{ Classification }} & Type & 1 & 4 \\
\hline & & & Shape (size, color) & - & - \\
\hline & & & Growth characteristics & 1 & 1 \\
\hline & & & Etc. & - & 1 \\
\hline \multirow{3}{*}{$\begin{array}{l}\text { Plants } \\
\text { information }\end{array}$} & \multicolumn{3}{|c|}{ Characteristics } & 1 & 3 \\
\hline & \multicolumn{3}{|c|}{ Cultivation \& harvest time } & - & 2 \\
\hline & \multicolumn{3}{|c|}{ Administration (water, fertilizer, disease and pest) } & 1 & 3 \\
\hline
\end{tabular}

Note. One domestic application and seven international applications were analyzed.

\footnotetext{
3) Out of 15 mobile applications for garden design developed in Korea and overseas, 8 programs were analyzed, excluding 7 applications on guidebooks for designing and creating gardens. (Since the purpose of this study was to provide basic data for developing mobile applications for garden design targeting users who actually intend to create gardens, the entire components of applications were analyzed based on actual spaces. However, since a large quantity of contents on plants such as methods for expression and classification and the existence of available information were included in virtual space-based applications, a total of 8 programs including actual space-based and virtual space-based programs together.)
} 
shed their design interface that distinguished species. In terms of the classification of plants, the number of those that classified plants by description was the highest (4). In terms of information on plants, 3 programs provided the characteristics of plants, and information on cultivation and harvest time ( 2 programs) and methods for maintaining plants ( 2 programs) was also provided.

Plants of which information was provided in the domestically-developed program for indoor garden design were divided into upper trees ( 7 species), intermediate trees (14 species), lower trees (31 species) and ground cover trees (18 species). The section of 'decorating with functional plants' suggested plants under 4 themes such as removing formaldehyde, improving indoor humidity, creating anions, and removing carbon dioxide. However, due to the characteristics of indoor gardens, there were limited species of plants available for indoor gardens, and 57 species (about 87.7\%) of the entire indoor plants (65 species, duplicated species excluded) were cultivated plants. There were only 8 species (about $12.3 \%$ ) were native plants. Meanwhile, overseas programs established services, other than the contents mentioned above, such as recommending suitable species of plants for users' conditions (climate, soil, sunlight, etc.), providing simulations of growing plants over time, and suggesting proper planting spacing depending on the area of planting.

The contents on plants in the currently-available mobile applications for garden design were found to provide not enough information necessary for those who actually tend gardens and cultivate plants such as the plant time and proper spacing of garden plants, combinations of plants and plant species recommended for each season, and recommended species by color or space. In particular, since there was no outdoor garden program in Korea, it was difficult to obtain the list of and information on plants in gardens in Korea, in particular, native plants in Korea, and thus it will be necessary to improve this area by developing new programs in the future.

\section{Suggestions for the future development of domestic mobile garden design applications}

The results of the survey and analysis of mobile applications for garden design showed that garden programs have been rapidly developed in various areas overseas, but that the number of mobile applications for garden design and the quantity of their information platforms in Korea were insufficient. It is not convenient for Koreans to use overseas programs due to language issues and different climatic conditions, plants and garden styles from those in Korea. In order to give practical help to users, it will be necessary to develop programs that can comprehensively cover a series of processes from planning and designing gardens to purchasing and installing products to maintaining gardens. In particular, since there is no program that contains information on gardens and plants suitable for users in Korea, a comprehensive garden application through which gardens are planned and maintained needs to be developed in Korea.

The currently available garden-related programs were comprehensively analyzed in line with the process of creating gardens, and it was found that mobile applications for garden design for users in Korea who actually intend to create gardens need to include contents such as providing other cases of gardening, guides for creating gardens and basic information on plants, suggesting space designs and simulations of designed gardens, and plants and materials suitable for users, providing methods for creating and maintaining gardens (harmful insects, fertilizers, watering, transplanting, etc.), inducing purchasing through links to suppliers' online sites, addressing the curiosity of users through counseling with experts, and establishing networks between users. In addition, the distribution and use of various garden plants can be promoted by suggesting widely-used plants in gardens, or providing information on plants by season and by color to allow users to choose as they like, and thus recommending suitable species of plants based on them, and connecting them with suppliers' online sites. A flowchart for mobile applications for garden design was established based on these components as shown in Figure 11. Figure 11 is a sample flowchart that contains necessary components for garden design applications 


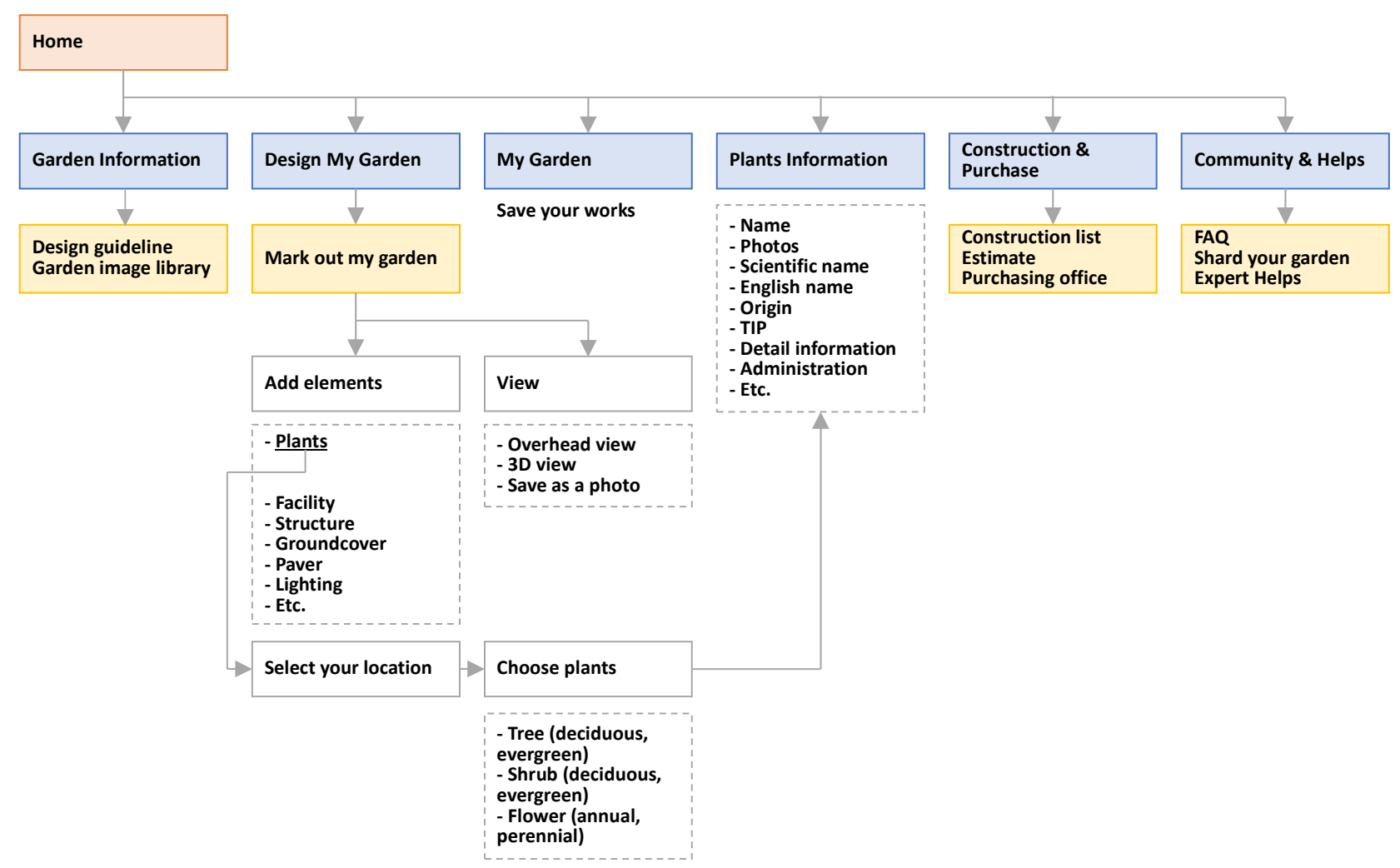

Figure 11. Suggested flowchart of Garden Design Mobile application.

suggested in this study, and thus the flowchart can be differently realized depending on the detailed components and contents of applications.

When developing mobile applications for garden design, marketing strategies and business models also need to be considered accordingly. Marketing strategies utilizing mobile applications continuously evolve into new forms to meet changes in marketing media, and 4R (Reflex, Reality, Real place, Real communication) strategies ${ }^{4}$ draw immediate reactions from users, and provide realistic experiences, information on the actual location of use, and real-time communications. The currently available mobile applications for garden design, utilizing garden design contents (design elements), allow users to formulate the design of gardens in real time and thus to immediately react to it, and induce users to experience real-size garden designs. However, they do not provide enough information connected with information on the actual location of use (selecting suitable species of plants considering regional characteristics), and interactions through real-time communications (sharing garden designs, exchanging information, counselling with experts, etc.). When developing applications for garden design, more expanded business models can be established by allowing users to exchange information with each other or with experts, and to interact with others, and thus by connecting them with suppliers of products related to gardens.

Revenue models of mobile applications can be divided into free models (free advertisement, advertisement-based models), paid models, and freemium models (Shin et al., 2011), and considering the active engagement of and interactions

4) Unlike mass marketing utilizing TV, recent online and mobile marketing strategies are centered on interactions with consumers. Internet marketing strategies replaced 4P (Product, Place, Price, Promotion) strategies with 4C (Contents, Cost to customer, Community, Connection) strategies (Han, 2003). Recently, 4R (Reflex, Reality, Real place, Real communication) strategies have been utilized in applications (Kim, 2010). 
between users in mobile applications for garden design, freemium models ${ }^{5)}$ seem to be suitable.

Recently, as garden culture has spread across the country in Korea, studies on gardens and plants have been actively conducted in various fields, and their results and findings are provided in various forms such as educational programs, publications, books and research institutes' websites. As part of the development of contents on garden culture, data of earlier studies can be utilized in applications through which research institutes can promote their organizations and research tasks, and improve the utilization of research results and findings. Users, at the same time, can access technical information more easily, and thus both research institutes and users can be benefited from the applications. In terms of the operation of applications, applications can be connected with the websites of suppliers through which suppliers can promote their products that are essential to create gardens such as plants, facilities and gardening products and increase their sales, and users can conveniently purchase products, increasing mutual interests. For this reason, this study suggests to develop mobile applications for garden design based on the freemium revenue model through which applications can provide users with services such as garden design programs, information on plants, counseling with experts, links to suppliers and contractors, and obtain profits from advertisement and sales commissions (Figure 12). To induce consumers' purchasing behaviors, the realistic experiences of garden designs can be expanded through graphics, and location-based services can lead to actual purchasing, which is expected to create a more effective revenue structure. In addition, it is expected to attract more users through promotion activities in various garden-related cultural events that have been held across the country.

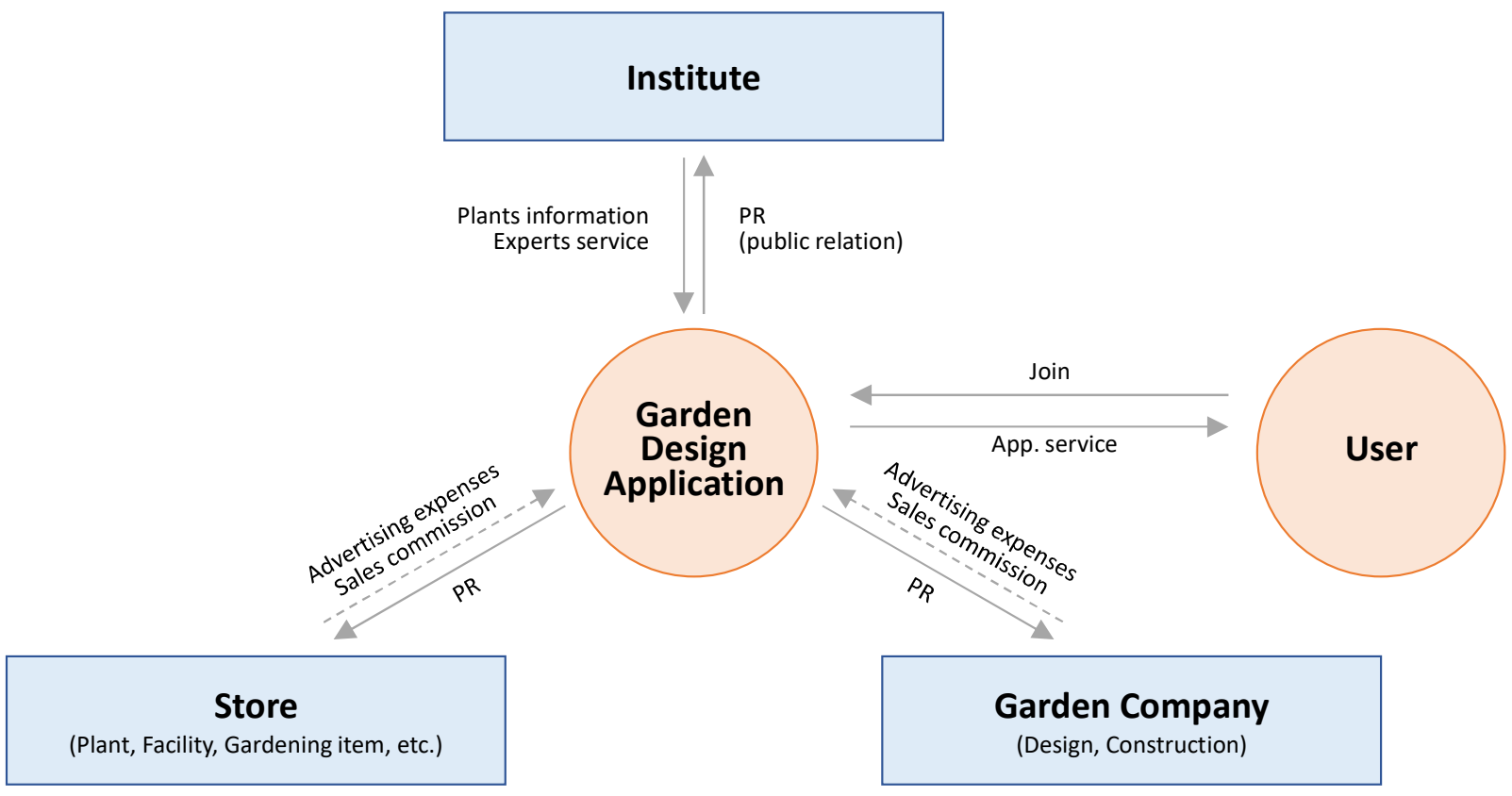

Figure 12. Garden design mobile application business flow. Solid-line arrows represent service flow and dotted arrows represent cash flow.

5) A freemium model, as a model that combines free and premium services, secures subscribers through free services, and seeks profits based on them. Basic services are provided for the users of free contents, and those who want to use more services need to pay for them (Lee, 2012). It is the most preferred business model for mobile applications, and the majority of the currently available mobile applications for garden design use the model. 


\section{Conclusion}

This study aimed to examine the current status of mobile applications for garden designs that have been developed in Korean and abroad, to extract contents essential for garden design programs, and thus to provide basic data that can be utilized in developing mobile applications for garden design going forward. To do so, among mobile applications that were registered on Google Play App Market in Korea and abroad as of July, 2018, free programs released either in Korean and English (1 application in Korea, 14 applications overseas) were analyzed. and they were largely divided into 'design programs based on real space,' 'design programs based on virtual space' and 'garden design and plan text books.' As this study aimed to analyze components of programs for those who actually intend to create gardens and to provide information that can be utilized in developing mobile applications for garden design going forward, this study focused on 'mobile applications for garden design based on real space (1 application in Korea, 3 applications overseas)' that can provide practical help for users, and their components and contents were analyzed.

The results of analysis showed that garden programs have been rapidly developed in various areas overseas, but that the number of mobile applications for garden design and the quantity of their information platforms in Korea were significantly insufficient. Against this backdrop, a flowchart for mobile applications for garden design was based on the results of the analysis of the existing garden design applications. The established flowchart in this study covers a series of processes from planning and designing gardens, to purchasing and installing products to maintaining gardens for users who actually intend to design and create gardens. Those who are interested in gardens, in particular, those who intend to create gardens will be able to design their own gardens, to collect information on plants and facilities required to create their gardens and to purchase them if they want through these applications. Users can also address issues they may confront while creating and maintaining gardens through counseling with experts. In addition, based on 4R (Reflex, Reality, Real place, Real communication) marketing strategies for mobile applications, a freemium-based revenue model was suggested. The realistic experiences of garden designs can be expanded through graphics, and the results and findings of studies on gardens and plants can be also provided. Consumers' purchasing behaviors can be induced by providing location-based services and establishing systems connected to actual facilities related to gardens and suppliers of plants. It is also expected to attract more users through promotion activities in various garden-related cultural events that have been held across the country.

\section{References}

Google Play Store. n.d. Retrieved from https://play.google.com/store/apps

Han, S.R. 2003. Customer orientational distribution strategy and application of internet marketing. Marketing 37(11):29-36. Retrieved from http://www.prideofkorea.com

Kim, Y.H. 2010. App Marketing. Seoul, Korea: Deonan.

Kwak, J.S. and J.M. An. 2013. A study of the core success factors for the development of application offshore. Entrue J. Inf. Technol. [LGCNS] 12(2):39-53.

Lee, D.J., H.Y. Roh, M. Kim, H.J. Cho, H.M. Lee, D.G. Cho, Y.J. Song, H.R. Cho, and J.H. Chon. 2017. Analysis of garden preferences of visitors to Korea Landscapes and Gardens Expo in 2017 - Focused on location and plant characteristics -. J. Korean Inst. Landsc. Archit. 45(6):126-136.

Lee, R.R. 2013. The study of the 'Healing Contents' based on smart applications. Master's thesis, Inha University, Incheon, Korea.

Lee, W.H. 2011. A study on the current status and invigoration plan of education contents for smart TV. Master's thesis, Yeungnam University, Gyeongsan, Korea.

Lee, Y.H. 2012. The present and prospect of mobile applications (No. 2011-20 Series 48). Naju, Korea: Korea Creative 


\section{Content Agency.}

Park, E.Y. 2010. A study on the design method of flowering plants used in the English White Gardens - Focusing on Sissinghurst, Barrington Court built in the early 20th century -. J. Korean Inst. Tradit. Landsc. Archit. 28(4):144-153.

Shin, Y.G., Y.H. Choi, H.J. Kim, S.G. Lee, H.S. Eom, M.J. Lee. 2011. Research on the method of promoting the platform and application industry. Gwacheon, Korea: Korea Communications Commission.

Song, E.J. 2012. A case of the mobile application system development using location based service. J. Digit. Contents Soc. 13(1):53-60.

Song, H.B. and A.Y. Kim. 2012. Study on status of planting herbaceous flowering plants and improvement in apartment complexes - Focused on the expert survey -. Proc. Korean Inst. Landsc. Archit. 2012(10):130-134.

Yang, H.J. and J.R. Lee. 2011. A study on the analysis of the GUI design of the applications used in the smartphone base. Treatise Plastic Media 14(2):107-116. Retrieved from http://www.illusart.kr

Yum, H.L. and S.H. Joo. 2017. A study on classification of garden styles by analyzing garden expo entries. J. Korean Soc. Flor. Art Des. 36:39-58. 